\title{
RIFLESSIONI SU PERCORSI SPIRITUALI ED ECCLESIALI DI BRASILIANI IMMIGRATI IN ITALIA
}

\author{
Reflections on spiritual and ecclesial paths of Brazilian immigrants in Italy
}

Nel mese di giugno 2017 il CSEM - Centro Scalabriniano di Studi Migratori, in collaborazione con la Fondazione Migrantes e la Provincia Europea delle Suore Missionarie Scalabriniane, ha realizzato uno studio tra le comunità di brasiliani nel contesto ecclesiale italiano.

La ricerca aveva come obiettivo conoscere e ascoltare degli immigrati brasiliani credenti che, arrivati in Italia, sviluppano strategie di riconfigurazione della fede ricevuta nel Paese d'origine, vivendo percorsi ed esperienze ecclesiali nuove in seno alla chiesa di immigrazione.

Sono state realizzate visite in loco nelle comunità e tra gli animatori o coordinatori di vari gruppi, dove è stato anche possibile partecipare ad attività sociali e pastorali. Sono state realizzate 35 interviste, coinvolgendo 60 persone, tra immigrati provenienti dal Brasile e italiani che assistono o interagiscono con i brasiliani credenti che partecipano alla realtà ecclesiale in Italia. Tutte le interviste sono state registrate in audio, per un ascolto più fecondo degli apporti dei protagonisti che vivono la migrazione con fede e la fede in emigrazione. In alcuni casi, la riflessione sull'esperienza della fede e del vissuto ecclesiale è avvenuta nella forma di un dialogo interattivo nei gruppi o nelle comunità frequentate regolarmente dai soggetti ascoltati, che si riuniscono per amicizia, fede o per solidarietà etnica ${ }^{1}$.

Sebbene la maggior parte dei partecipanti alla ricerca frequentava gruppi di connazionali, alcuni si facevano presenti, allo stesso tempo, anche in strutture ecclesiali territoriali italiane e altri erano inseriti unicamente

Centro Scalabriniano de Estudos Migratórios - CSEM. Brasília - DF, Brasil. E-mail: carmem.lussi@ gmail.com. Orcid: 0000-0002-5666-7870.

1 Coloro che hanno partecipato allo studio erano residenti nelle province di Padova, Treviso, Mantova, Verona, Asti, Torino, Piacenza, Bergamo, Roma, Firenze, Milano, Brescia e Catanzaro. I brasiliani ascoltati sono originari di 12 Stati del Brasile: Rio Grande do Sul, Santa Catarina, Paraná, São Paulo, Mato Grosso do Sul, Rio de Janeiro, Espírito Santo, Brasília, Minas Gerais, Maranhão, Bahia e Paraíba. 
nel tessuto ecclesiale locale, senza legami con aggregazioni di brasiliani. Indipendentemente dallo specifico di ogni contesto locale, "la missione della chiesa nell'ambito della mobilità umana può essere descritta come una missione tra, per e con i migranti, in cui ognuna delle preposizioni indica un aspetto particolare della missione. II 'tra' indica il luogo della missione, il 'per' ne sottolinea i destinatari, e il 'con' evidenzia la necessità di includere questi ultimi nella missione stessa" (Baggio, 2018, p. 9).

\section{Tratti specifici di un'eredità spirituale ed ecclesiale}

Le comunità di immigrati che si riuniscono per nazionalità (o lingua) di origine sono un riferimento identitario importante, in cui convergono fede, tradizione ecclesiale e cultura in un insieme inscindibile. Nel caso dei brasiliani, gli immigrati arrivano da un Paese caratterizzato da dimensioni continentali e da una forte pluralità culturale ed ecclesiale.

L'accoglienza delle comunità territoriali italiane aiuta a superare le fatiche del processo migratorio e può fare scoprire la ricchezza del bagaglio culturale, ecclesiale e spirituale di tanti immigrati, aiutandoli a 'sentirsi a casa', nonostante le differenze e l'estraneità del nuovo contesto ecclesiale. Talvolta prevale il bisogno di "celebrare nel nostro modo brasiliano, per cantare i nostri canti che ci piacciono, per state con altri brasiliani che hanno la nostra stessa esperienza e la stessa nostalgia... e un poco anche per condividere con altri la nostra gioia brasiliana delle comunità cristiane" ${ }^{\prime 2}$.

Interrogati sui tratti specifici dell'esperienza di fede in Brasile e il modo di vivere la Chiesa nel Paese di origine attraverso lo sguardo dell'emigrante che non vuole abbonare la fede, i brasiliani che hanno partecipato alla ricerca hanno individuato alcune caratteristiche del vissuto credente e del modo di accogliere, trasmettere, celebrare e vivere la fede e la Chiesa in Brasile di cui ne sentono più fortemente la mancanza in emigrazione. Gli intervistati indicano alcuni tratti propri dell'eredità cristiana brasiliana con la quale sperano e credono di poter arricchire la Chiesa in Italia. Ai fini di questo breve relato, sottolineo tre aspetti: l'animazione liturgica, la relazione fede-vita e il tema del laicato.

\subsection{Animazione liturgica}

L'animazione liturgica è il tratto della Chiesa brasiliana di cui gli immigrati di quella terra sentono di più la mancanza quando sono in Italia. Gli intervistati manifestano amarezza nel 'dover andare a messa' dove la celebrazione eucaristica non ha i tratti del calore umano, della vitalità, vicinanza e interrelazione tra i partecipanti che molti di essi erano abituati a vivere in Brasile. Mancano i canti con ritmi, suoni e testi che fanno riferimento alla vita

\footnotetext{
2 Intervista n. 4, brasiliana, Torino.
} 
della gente; mancano la luce, il calore e i colori dei luoghi sacri, che in Italia sono 'freddi, bui e spesso anche maleodoranti'; lascia perplessi che i lettori che proclamano le letture siano sempre gli stessi e che la stessa persona legge letture e salmo responsoriale, un giorno sì e l'altro anche; demotiva il fatto che ogni persona presente sia percepita come un numero in chiesa, senza relazioni, con un saluto della pace frettoloso e senza incontrare lo sguardo.

In terra straniera, la preghiera è qualcosa che, spesso, diventa per i migranti un'ancora di sostegno e punto di riferimento nel cammino, per cui, frequentare le chiese e, specialmente, partecipare all'Eucaristia si trasforma in qualcosa di fondamentale per la fede dei migranti (Lussi, 2016, p. 245-252). Così, gli elementi della liturgia che divergono tra il Paese di origine e il contesto locale in emigrazione possono suscitare un disagio, che può cambiare notevolmente nel corso del temo, quando il processo migratorio entra nelle tappe più avanzate dell'inserimento nel Paese scelto per vivere.

Il rinnovamento liturgico, che non ha prodotto rituali nuovi in Brasile, ha tuttavia promosso adeguazioni del canto e delle preghiere, di gesti e di simboli che hanno favorito, ampiamente, la partecipazione dei credenti in modo attivo e coinvolgente alla liturgia e incoraggiato la relazione diretta della celebrazione della fede con la vita delle comunità. E di tutto questo i credenti brasiliani ne sentono la mancanza in Italia, dove non percepiscono aspetti della vita della gente nella celebrazione dei sacramenti, specialmente dell'Eucaristia. La loro percezione è che manca vitalità, non solo nella liturgia, ma anche nell'esperienza ecclesiale in generale.

La centralità della liturgia per la vita cristiana e della vita dei credenti nella liturgia appare nella nostalgia dei migranti brasiliani come incapacità di celebrare, dove la celebrazione non comporta l'inclusione della loro vita e l'estraneità propria del percorso migratorio appare come un essere stranieri nella propria Chiesa.

Ci sono due momenti in cui mi rendo conto che non sono in Brasile: quando vado a messa e quando sono nella messa. Qui la messa è tutto di corsa, senza spazio per la vita... è la struttura parrocchiale in Italia che è molto chiusa e difficilmente dà spazio affinché gli immigrati possano entrare e diventare Chiesa ${ }^{3}$.

Oltre all'animazione liturgica, un altro aspetto della Chiesa brasiliana riguardante la preghiera di cui gli immigrati brasiliani sentono nostalgia è il fervore che motiva alla partecipazione in momenti celebrativi della comunità e la preghiera nelle famiglie, gli "incontri"4, specialmente quelli tra vicini di casa

3 Intervista n. 35, brasiliano, Padova.

4 La parola encontros, che appare spesso tra gli elementi di nostalgia che i brasiliani si portano dentro nei percorsi migratori all'estero, si riferisce a un'ampia gamma di tipi di attività, in cui persone e famiglie si ritrovano, spesso nelle famiglie, ma anche negli ambienti comunitari, dove si coltivano le relazioni, l'amicizia e la formazione religiosa e spirituale, se non anche la formazione 
per il rosario e altri incontri che, in molte diocesi brasiliane, si fanno tra vicini di casa per trattare temi di interesse comune o per l'ascolto della Parola di Dio.

Gli intervistati sentono che il fervore e il coinvolgimento nella vita di fede e nella preghiera è meno significativo in Italia, dove considerano che " $c$ 'è meno fede, meno fervore e poca partecipazione, persino nella Messa domenicale, dove vanno quasi solo anziani" ${ }^{\prime \prime}$.

\subsection{Relazione fede-vita}

Un secondo aspetto molto forte nella percezione della specificità del modo brasiliano di essere credenti e di essere chiesa emerso dalla ricerca è la relazione fede-vita. Molti brasiliani lamentano, soprattutto nel primo approccio con il contesto ecclesiale italiano, la percezione di una fede rituale, talvolta morale, ma soprattutto distaccata dalla vita concreta in cui vivono i fedeli, lontana dalla realtà sociale, culturale, storica che la gente vive.

Da noi, in Brasile, quello che più sento come diverso da qui è la relazione fedevita: da noi la fede è profondamente collegata alla vita della gente, si celebra quello che si vive; si manifesta la gioia; la liturgia è collegata con la vita e con i problemi sociali e politici, perché la fede è intrecciata con tutto ciò che è la persona, la stessa persona che vive in società, che lavora e che soffre le conseguenze del suo contesto e Dio è presente in tutto questo ${ }^{6}$.

Nonostante sia evidente che anche in Brasile ci sono "tanti battezzati non evangelizzati, cristiani indifferenti nella realtà quotidiana che cooperano in maniera negativa ad ampliare la frattura tra vita e fede" (Valero Cárdenas, 2014, p. 433), gli immigrati brasiliani cattolici ascoltati in Italia sottolineano una povertà spirituale che identificano come mancanza di quella relazione della fede con la vita e di quella penetrazione della vita nelle espressioni e nei momenti in cui la fede si nutre, si esprime e si celebra.

La relazione fede-vita appare per i brasiliani nella capacità - che talvolta diventa necessità -, di rapportare la fede, i temi della spiritualità e della vita ecclesiale alla quotidianità e agli episodi della vita privata delle persone, "perché la nostra è una fede che si configura come una relazione con un Dio che si è fatto uomo e che non chiede altro della sua sequela, che una relazione d'amore che penetra tutte le dimensioni della vita dei credenti" ${ }^{\prime \prime}$.

Questa dimensione di fede nella vita, normalmente, diventa una chiara

sensibilità dei cristiani per le tematiche del sociale, per i problemi che affliggono la popolazione o anche impegno nella politica, piuttosto che nel sindacato o nelle organizzazioni per i diritti umani, che è qualcosa che in Italia sta un po' scemando.

e l'informazione su temi sociali e culturali, su diritti umani e cittadinanza responsabile, e dove si moltiplica la solidarietà, si sostiene la fede e si cresce nella preghiera e nella vita comunitaria.

5 Intervista n. 6, brasiliana, Torino.

6 Intervista n. 36, brasiliano, Roma.

Intervista n. 36, brasiliano, Roma. 
(...) Questa è un'apertura mentale e una testimonianza di fede che la comunità brasiliana offre all'Italia e che ci porta speranza per la nostra realtà italiana che ancora resiste in questo senso $^{8}$.

La relazione fede-vita di cui gli immigrati cattolici brasiliani sono portatori e allo stesso tempo sono desiderosi di coltivare e riscoprire in Italia va incontro e risponde alla dimensione sociale dell'evangelizzazione che papa Francesco ricorda e raccomanda nella Evangelii Gaudium.

Forse il maggior contributo che la Chiesa brasiliana offre all'Italia, attraverso i suoi emigrati che scelgono questa terra come Paese di elezione, non sta tanto nelle comunità che possono costruire, ma nella qualità e nei tratti della testimonianza di una fede che può essere vissuta in un altro modo, una fede legata alla vita, che determina le relazioni interpersonali, che penetra in tutti gli ambiti dell'esistenza, che diventa qualità di impegno nelle relazioni con gli altri, capacità di leggere con speranza le difficoltà e i fatti di tutti i giorni.

\subsection{Laicato responsabile e attivo}

Una terza caratteristica della chiesa brasiliana che arriva in Italia attraverso le migrazioni sono i credenti, in particolare, i laici impegnati, molte volte formati in comunità vive e dinamiche, che esprimono un laicato responsabile e attivo, erede di un modo di essere Chiesa dove i laici prendono l'iniziativa, molto di più che in Italia, e dove la collaborazione e l'interazione con il clero è, normalmente, molto più leggera e intensa, ad un tempo.

Grandi parrocchie, spesso con delle comunità lontane dalla sede, esigono dalla Chiesa brasiliana la formazione e la fiducia nei catechisti e negli animatori di comunità, come guide della liturgia e della festa, del servizio della carità e dello sviluppo locale, così come dell'organizzazione e della gestione delle finanze per l'autosostentamento delle comunità e del loro clero. Dalla Chiesa brasiliana I'Italia può anche imparare a "valorizzare e riconoscere il potenziale della vita religiosa femminile, della competenza e capacità della vita religiosa di costruire la Chiesa con la loro specificità e modo proprio di assumere la missione, perché la Chiesa non è solo il clero; la chiesa è anche femminile e laicale..." ${ }^{\prime 9}$.

Molti immigrati cattolici provenienti dal Brasile si meravigliano nel constatare che in Italia i laici assumono prevalentemente funzioni di assistenza e aiuto puntuale ai parroci, con poca possibilità e capacità di iniziativa, salvo per attività alternative alla vita della comunità cristiana. La proattività dei laici con certa maturità cristiana ed ecclesiale è una risorsa che arricchisce le comunità cristiane.

Considerando che gli immigrati brasiliani raramente si trovano tra $\mathrm{i}$ soggetti o gruppi destinatari dei servizi emergenziali rivolti agli immigrati del tipo 'attività caritatevoli', spesso diventano estranei o sono considerati assenti nei

8 Intervista n. 33, italiano, Bergamo.

9 Intervista n. 35, brasiliano, Padova. 
contesti locali. Eppure, è proprio andando oltre all'emergenza che si possono incontrare e conoscere gli immigrati dal Brasile, i quali si aspettano dall'Italia cristiana e cattolica quella reciprocità propria della fede e della vita ecclesiale, "I'intercambio, la relazione umana paritaria tra i membri della comunità e i migranti" (Campese, 2015, p. 89).

I brasiliani che in patria erano laici impegnati, una volta arrivati in Italia si aspettano generalmente un'accoglienza che valorizzi il loro percorso, che spesso però è disattesa, e la delusione non sempre è elaborata con quella misericordia che fa andare avanti senza remore. Eppure, laddove gli alti e bassi della prima ora vengono superati, specialmente tra i cristiani con maggiore formazione e cammino spirituale, si ascoltano espressioni di stima e di valorizzazione di tanti aspetti della Chiesa che si trova in Italia che hanno saputo apprendere e assumere per sé stessi e per le loro famiglie, nel percorso di reinvenzione e reinterpretazione dei vissuti cristiani, durante l'esperienza migratoria.

Nella riflessione su cosa apportano alla Chiesa in Italia, gli immigrati fanno riferimento al proprio vissuto spirituale, alla fede che li anima e anche alla "fede che mi ha animato a lasciare la mia terra confidando in Dio, come altrimenti avrei potuto partire? Sono venuto qui con la fede e per la grazia di Dio e questa fede è la mia forza e il senso del mio restare qui e fare del mio meglio per vivere bene in questa terra"10. Gli immigrati brasiliani, insieme con tante altre comunità cattoliche immigrate

stanno diventando essi stessi i protagonisti per eccellenza della missione con la loro fede semplice, ma allo stesso tempo profonda, tenace e ricca di espressioni popolari. In Italia è sufficiente partecipare alle celebrazioni e festività delle comunità [immigrate]... per rendersi conto della profondità umana, della vitalità della fede, della bellezza delle liturgie, della generosità solidale e dell'energia evangelizzatrice che [gli] immigrati stanno apportando alle chiese locali nelle quali sono presenti (Campese, 2015, p. 93).

\section{Percorsi spirituali ed ecclesiali promissori}

L'ascolto di immigrati brasiliani credenti sull'esperienza spirituale ed ecclesiale vissuta nel percorso migratorio rivela due percezioni prevalenti. Alcuni si aggrappano alla fede come a un'ancora sicura, astraendola dalle sue caratteristiche culturali e contestuali, persino dalla lingua, e sottolineando il fatto che la liturgia e, soprattutto, la preghiera trascendono la contingenza del luogo e del contesto e rimangono come riferimenti di significato anche in terra straniera. Altri, che sono la maggioranza, da un lato registrano lo smarrimento del primo impatto all'arrivo in Italia e dei primi contatti con la Chiesa italiana, normalmente attraverso la liturgia eucaristica, e, dall'altro lato, raccontano la consolazione quando c'è stata la possibilità di 'ritrovare' il Brasile nelle

${ }_{10}$ Intervista n. 16, gruppo della comunità cristiana degli immigrati dal Brasile, Verona. 
celebrazioni della fede e della festa in gruppi e comunità cristiane di brasiliani in Italia (Lussi, 2016).

Tra gli immigrati ci sono credenti che non si misurano con il contesto ecclesiale italiano e decidono di vivere e curare la propria fede esclusivamente nella (se possono contare su una) comunità cristiana brasiliana che si ritrova per celebrare la fede e organizzare incontri ed eventi religiosi e culturali propri della terra d'origine. La maggior parte di quanti partecipano alle comunità di brasiliani in Italia si inserisce nei contesti ecclesiali italiani, ma non rinuncia a vivere e a condividere con altri connazionali momenti periodici di convivenza e di celebrazione della fede e della vita tra connazionali. Altri ancora, credenti nel Paese d'origine, vivono inseriti nei contesti ecclesiali italiani, spesso invisibili, più o meno attivi nelle parrocchie dove abitano.

Le fatiche del percorso per diventare chiesa con la Chiesa locale è reciproca, sia dalla parte degli stranieri, sia dalla parte degli italiani, seppur con sfumature diverse. Alla disponibilità e dedizione nell'accoglienza aperta da parte degli italiani, corrisponde la disponibilità, l'apertura e lo sforzo di andare incontro, da parte degli immigrati.

Ci riuniamo per celebrare nel nostro modo brasiliano di vivere la fede e questo è anche una testimonianza per gli italiani, però partecipiamo delle comunità italiane, perché insieme impariamo gli uni dagli altri, così la nostra fede cresce anche in terra straniera e scopriamo/apprezziamo il modo italiano di vivere e di credere $^{11}$.

\section{Bibliografia}

BAGGIO, Fabio. Marta, Marta, tu ti preoccupi e ti agiti" (LC 10,41). L'ascolto itinerante nella progettazione della pastorale migratoria. Lido di Ostia, 20/11/2018. Disponibile in <https://migrants-refugees.va/it/wp-content/ uploads/sites/3/2018/11/Contributo-Baggio.pdf $>$.

CAMPESE, Gioacchino. "Non di solo pane..." (Mt 4, 4). Missione della Chiesa dei e con i migranti. In: MAZZOLINI, Sandra. Evangelizzare il sociale. Prospettive per una scelta missionaria. Città del Vaticano: Urbaniana University Press, 2015. LUSSI, Carmem. Migrações e alteridade na comunidade cristã. Ensaio de teologia da mobilidade humana. Brasília: CSEM, 2016.

LUSSI, Carmem. Incontro che trasforma. Sfide e opportunità della relazione tra chiesa italiana e le migrazioni. Quaderni Migrantes. Todi: Tau \& CSEM \& Fondazione Migrantes, 2018.

VALERO CÁRDENAS, Yolanda. I laici nella Chiesa d'America Latina e modelli di comunità. In: TREVISIOL, Alberto (a cura di). In ascolto dell'America. Popoli, culture, religioni, strade per il futuro. Città del Vaticano: Urbaniana University Press, 2014, p. 431-443.

$\overline{11}$ Intervista n. 16, gruppo della comunità cristiana degli immigrati dal Brasile, Verona. 